\title{
Percutaneous hepatic perfusion with melphalan for unresectable liver metastasis
}

\author{
Humair S. Quadri ${ }^{1}$, Eden C. Payabyab ${ }^{1}$, David J. Chen ${ }^{1}$, William Figg ${ }^{2}$, \\ Marybeth S. Hughes ${ }^{1}$
}

${ }^{1}$ Thoracic and Gastrointestinal Oncology Branch, National Cancer Institute, Bethesda, MD 20892, USA.

${ }^{2}$ Genitourinary Malignancies Branch, National Cancer Institute, Bethesda, MD 20892, USA.

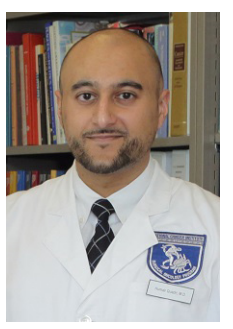

Humair S. Quadri, M.D., is a general surgery resident at Georgetown University Hospital in Washington, D.C. who completed a two year surgical oncology fellowship at the National Cancer Institute of the National Institutes of Health in Bethesda, Maryland. He received his M.D. from Georgetown University School of Medicine in Washington, D.C.

\begin{abstract}
Percutaneous hepatic perfusion (PHP) is an investigative technique for treating patients with diffuse unresectable metastatic liver disease. The technique has been clinically evaluated and shows great treatment potential for regional therapy to the liver. The advantage of PHP lies in its minimally invasive approach and ability to be repeated when compared to isolated hepatic perfusion. In a literature search, 135 publications were screened and 16 of these publications, including clinical trials and reviews, contributed to this review of PHP with melphalan. Melphalan is an alkylating agent that, when used as the chemotherapeutic agent in PHP, has shown potential for significant control of tumor burden in the liver, especially in metastatic ocular melanoma. In the current landscape of liver directed therapy, PHP is a viable option for those with unresectable metastatic disease to the liver. This article will focus on the technical aspects of PHP and describe the current data available from clinical trials, including outcomes of patients treated with this minimally invasive approach.
\end{abstract}

Key words: Percutaneous hepatic perfusion; melphalan; unresectable liver metastasis; metastatic melanoma to the liver; ocular melanoma

\section{Corresponding Author:}

Dr. Marybeth S. Hughes, Thoracic and Gastrointestinal Oncology Branch, National Cancer Institute, Room 4-5940, Building 10 - Hatfield CRC, Bethesda, MD 20892, USA. E-mail: hughesm@mail.nih.gov

Received: 24-06-2016; Accepted: 28-06-2016

\section{INTRODUCTION}

\section{What is percutaneous hepatic perfusion}

The treatment of metastatic disease to the liver is an evolving paradigm that has been evaluated with increasing potential over the past few decades. Though there are treatment options for solitary or localized liver lesions, there is no treatment consensus when multiple metastatic lesions are found throughout the liver. ${ }^{[1]}$ It is estimated that approximately $80 \%$ of people with liver metastasis are

\begin{tabular}{|l|c|}
\hline \multicolumn{3}{|c|}{ Access this article online } \\
Website: & Quick Response Code \\
http://www.hrjournal.net/ & \\
DOI: &
\end{tabular}

considered unresectable due to excessive tumor burden, tumor location, effect on inflow or outflow, an insufficient liver remnant, or a significant comorbidity. ${ }^{[2]}$ Most patients with liver-only unresectable metastatic disease have options of directed treatment. Percutaneous hepatic perfusion (PHP) is one of these novel techniques for patients with diffuse liver-only metastatic disease.

PHP is a minimally invasive procedure which allows for regional therapy to the liver. Arterial cannulation of the

This is an open access article distributed under the terms of the Creative Commons Attribution-NonCommercial-ShareAlike 3.0 License, which allows others to remix, tweak, and build upon the worknon-commercially, as long as the author is credited and the new creations are licensed under the identical terms.

For reprints contact: service@oaepublish.com

How to cite this article: Quadri HS, Payabyab EC, Chen DJ, Figg W, Hughes MS. Percutaneous hepatic perfusion with melphalan for unresectable liver metastasis. Hepatoma Res 2016;2:197-202. 
hepatic artery via a femoral artery puncture is used to selectively administer an anti-neoplastic agent directly to liver tumors. By endovascular venous cannulation, a unique double balloon catheter (Delcath catheter) is inserted into the inferior vena cava (IVC) to capture the hepatic venous outflow from the liver. Using veno-venous bypass, the chemotherapy laden blood can be captured at the hepatic vein confluence and filtered before returning to the systemic circulation by a central venous line. This novel treatment technique has evolved from original operative liver isolation techniques, which capitalized on the hepatic anatomy for inflow and surgical outflow control in liver directed perfusion. ${ }^{[3]}$

\section{History and development}

The first use of hepatic perfusion was reported by Dr. Robert Ausman in 1961 as a surgery resident at the Roswell Park Cancer Institute where he developed the technique. His initial studies were performed on animal models, and once the technique was standardized it was tested on 5 patients with different types of hepatic malignancies. Though there was no long term follow-up and significant toxicity noted with the procedure, there was a therapeutic effect described in 2 patients. ${ }^{[3]}$ This initial study helped lay the foundation for isolated hepatic perfusion (IHP) which has been refined over 60 years. Multiple centers have evaluated IHP with various chemotherapy agents, various tumor histologies, hyperthermic perfusion, and improved techniques. ${ }^{[4]}$

With data from isolated limb perfusion by Lienard et al..$^{[5]}$ in 1992, melphalan was initially tested in combination with tumor necrosis factor alpha (TNF $\alpha$ ). This regimen was used for IHP to treat liver disease. Early results at the National Cancer Institute showed a 75\% radiographic response rate with this combination and no diminishment of antitumor activity with advanced disease burden in the liver ${ }^{[3]}$ However, due to the unavailability of TNFa for continued clinical testing in the United States, melphalan has been the most widely used chemotherapeutic agent in current trials. Through these early studies of the operative technique for IHP, key elements and principles were noted and carried over to the minimally invasive PHP technique in use today.

PHP was initially reported approximately 20 years ago by 2 centers. The largest study described by Ravikumar et al. ${ }^{[6]}$ involved 28 patients who were treated with escalating doses of doxorubicin or 5-fluorouracil. Through the catheter based approach, the chemotherapy was administered via a hepatic artery catheter and collected and filtered using veno-venous bypass from the venous outflow of the liver. Concurrently, a phase I study by Curley et al. ${ }^{[7]}$ was being performed in patients with hepatocellular carcinoma. Similar to the early use of IHP, no long term follow-up data was published and these studies were not continued at these centers. However, these studies described the potential use of this procedure and contributed to the refinement of its technical feasibility.

In 2005, the comprehensive evaluation of PHP was conducted as a phase I trial at the National Cancer Institute where 28 patients were treated with melphalan PHP, for 74 treatments in a dose escalation format. The overall radiographic response rate was observed to be $30 \%$ (RECIST criteria), with rates as high as $50 \%$ in 10 patients with metastatic ocular melanoma. Though transient hepatic toxicity and some hematologic toxicity were observed, this study helped determine the maximum tolerated dose of melphalan $(3.0 \mathrm{mg} / \mathrm{kg})$ and established the groundwork for a multicenter trial. ${ }^{[8]}$ After

\section{Percutaneous Hepatic Perfusion}
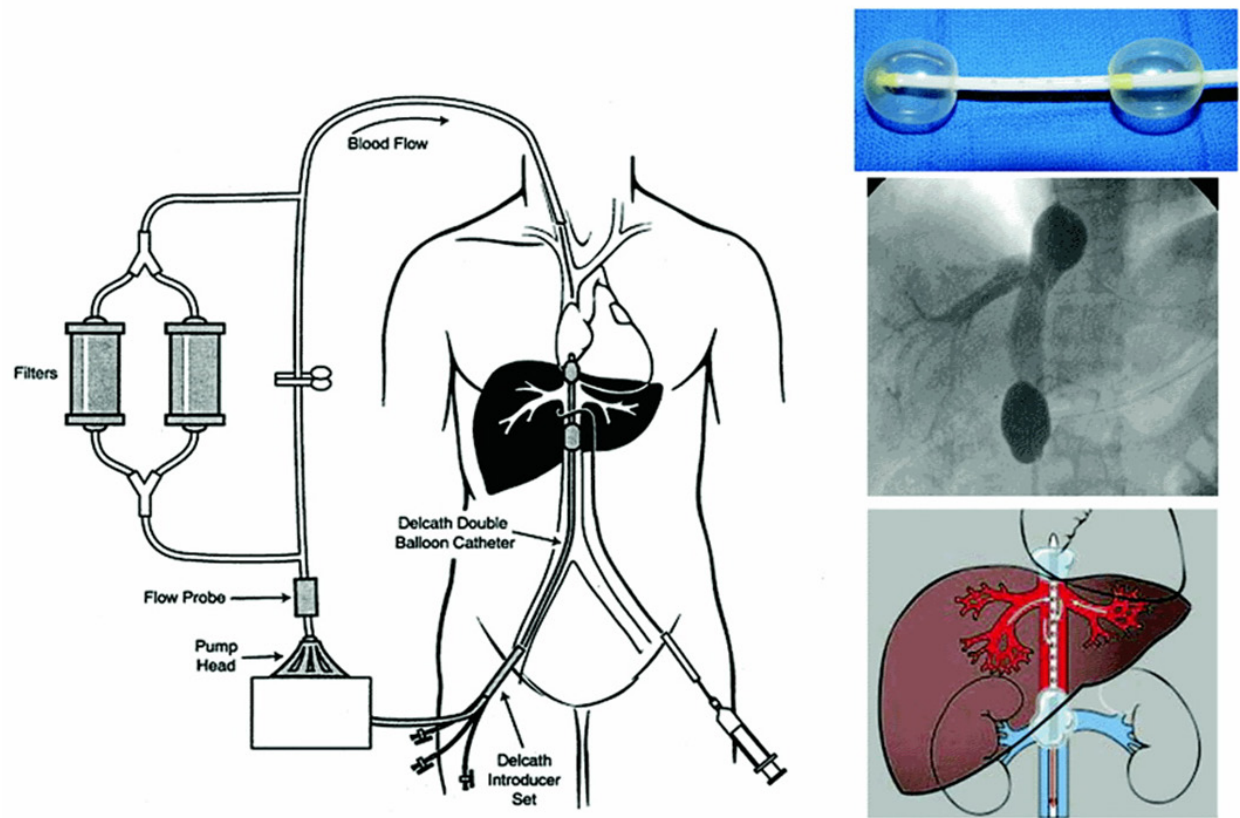

Figure 1: Diagram of the percutaneous hepatic perfusion system. This Delcath ${ }^{\circledR}$ Catheter System is used to infuse melphalan into the hepatic artery percutaneously (syringe) via the femoral artery. A double balloon catheter (shown in the upper right) is placed in the retro-hepatic inferior vena cava under fluoroscopic guidance (middle right image) to isolate the hepatic venous outflow. The multiple fenestrations along the balloon catheter then draw out the isolated blood which then is directed into the extracorporeal system. The blood is then pumped thorough a pair of activated charcoal filters, which extract the melphalan, before being returned to the systemic circulation. (This image has been reproduced with permission and purchase from The Cancer Journal) 
publication of this phase I data, a multi-institutional phase III random assignment control trial was started in 2005, where PHP with melphalan was compared with the current best available care (systemic chemotherapy, embolization, supportive care) in patients with metastatic melanoma with the majority of tumor contained in the liver. ${ }^{[3]}$ This trial was completed in 2010 and the results have recently been published, with analysis showing an increase in hepatic progression-free survival in the melphalan PHP arm compared to the best available care. ${ }^{[9]}$ Currently, there are numerous centers throughout the world evaluating PHP and improving the technical aspects and treatment outcomes.

\section{Evaluation}

We evaluated data using previous publications on methods of liver perfusion, ranging from reviews to clinical trials. An initial PubMed search with the keyword "percutaneous hepatic perfusion" was performed yielding 135 publications. Publications were excluded if they were not in English, had no mention of liver metastasis or liver tumors, or were not available online or through an easily accessible source. We then screened 25 publications relating to PHP using the addition of the keyword "melphalan". This search yielded 17 publications, only those that linked or contained primary data relating to PHP or IHP were selected, and ultimately 16 publications contributed to this review.

\section{TECHNICAL ASPECTS}

\section{Procedure}

As mentioned previously, PHP is a technique where a chemotherapeutic or biologic agent is delivered via catheterization of the hepatic artery. The hepatic venous
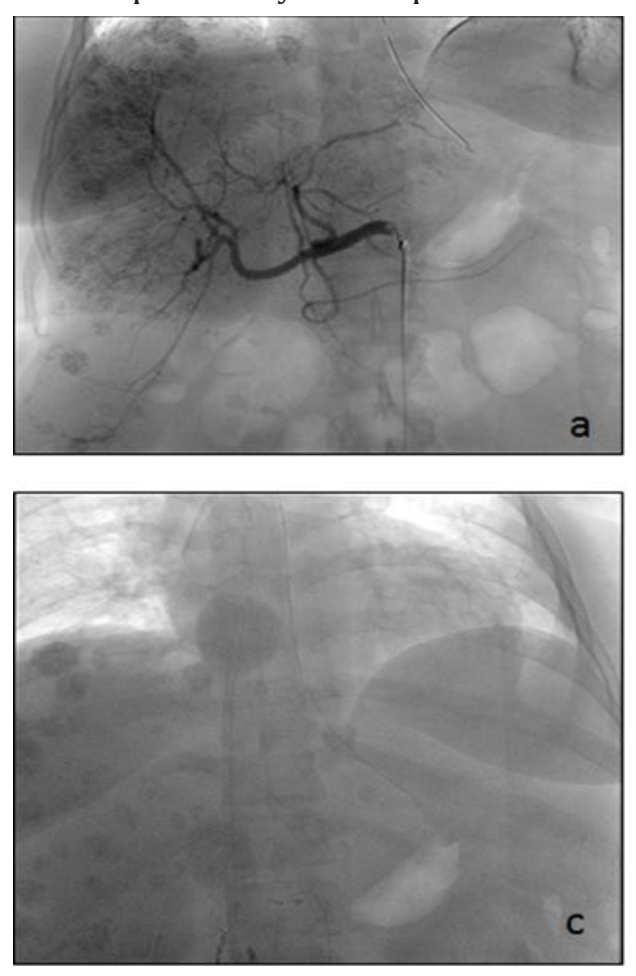

Figure 2: A 51-year-old female with a history of pancreatic neuroendocrine tumor and metastatic disease to the liver. (a) Common hepatic artery cannulated and filled with contrast defining the vascular anatomy of the liver. Visible are the numerous metastatic lesions which are contrast enhancing; (b) gastroduodenal artery

coiled after contrast evaluation; (c and d) intra-procedural images of hepatic venous system isolation

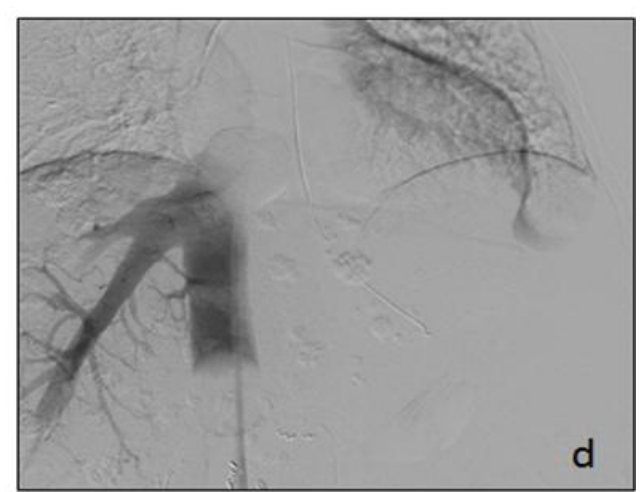

circulation is isolated via a special patented double balloon catheter directed via venous cannulation and fluoroscopically guided placement in the IVC (Delcath Catheter Systems, Delcath Inc., New York, NY). This allows for capture of the chemotherapy-laden effluent from the liver, which is filtered via veno-venous bypass prior to returning to the systemic circulation. ${ }^{[3,8]}$ PHP takes advantage of the tumor blood supply in which $90 \%$ of the tumor is supplied by hepatic artery inflow. In contrast, normal hepatocytes receive over $50 \%$ of their blood flow from the portal venous inflow. By isolating the hepatic arteries, infusion of chemotherapeutic agents are able to take the most direct circulatory pathway to liver tumors while somewhat sparing normal hepatocytes. It is critical to ensure that flow is isolated to the liver to avoid inadvertent chemoperfusion of non-target organs. Once the agent has completed its hepatic circulation, it is collected via fenestrations situated between patented double balloons of the catheter, from the hepatic veins as it enters the IVC. This catheter is initially placed and tested under fluoroscopy in the retrohepatic IVC so that the balloons are carefully seated cephalad and caudad to the hepatic veins. The blood is then directed through an extracorporeal filtration system (containing activated charcoal filter cartridges) which removes the agent prior to return to the systemic circulation via an internal jugular venous catheter [Figure 1].

The procedure is usually performed using general anesthesia with arterial line access placed for blood pressure monitoring, as well as internal jugular venous access for infusion from the veno-venous bypass circuit. The extracorporeal pump is primed with normal saline, and during the procedure, heparin is administered to maintain an activated clotting time at therapeutic levels. Percutaneous access of the right common

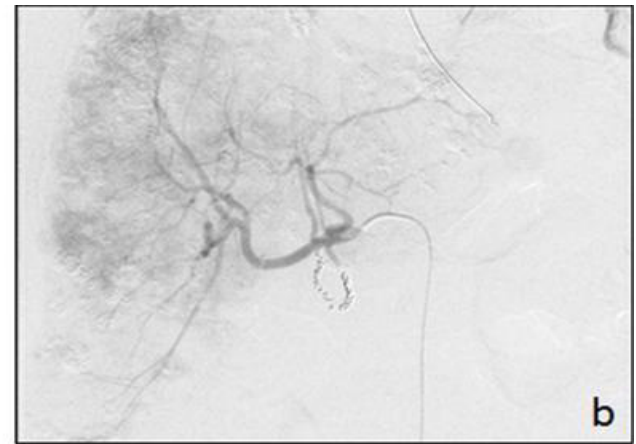

b 
femoral artery and vein are obtained, and an angiogram is performed via the celiac and superior mesenteric arteries to define the arterial anatomy. The gastroduodenal artery is usually embolized to minimize any extrahepatic perfusion and a catheter is positioned in the hepatic artery proper under fluoroscopic visualization. A double balloon catheter is then introduced percutaneously via the right common femoral vein. The cephalad balloon is inflated with contrast until it is maximally inflated while in the right atrium and then withdrawn until indentation of the diaphragmatic hiatus is visualized under fluoroscopy. The caudal balloon is then inflated until the balloon wall is deformed indicating a seal. Hepatic venous isolation is obtained both superiorly and inferiorly to the hepatic veins. Given the IVC will be blocked by the balloon, a bypass circuit is needed. The bypass circuit is composed of a venous Delcath $16 \mathrm{~F}$ polyethylene catheter with one large fenestrated lumen and 3 accessory lumens, flushed bypass tubing, 2 filters, and the internal jugular central venous return line. Contrast is injected via the fenestrated lumen to confirm that the hepatic outflow is sealed and there is no leakage of hepatic outflow into the systemic circulation. All of this is critical to be accomplished prior to administration of any chemotherapeutic agents. Since venous return from the lower extremities is blocked at this time, veno-venous bypass is initiated. Just prior to initiation of this bypass circuit and filter activation, some patients can experience a transient drop in blood pressure requiring additional fluid and infrequent vasopressor support.

After confirmation of vascular outflow isolation, chemotherapy is given for a $30 \mathrm{~min}$ continuous infusion via the proper hepatic artery catheter. Occasionally due to anatomy, the chemotherapy infusion must be split between the right and the left hepatic artery to avoid any chemotherapy infusion to organs other than the liver. The filtration circuit is continued for an additional $30 \mathrm{~min}$ after the chemotherapy is infused to ensure adequate removal of the agent. Reversal of anticoagulation after the procedure is achieved via protamine administration, along with fresh frozen plasma, as necessary for safe catheter removal. After the start of reversal, the balloons are deflated and the IVC and hepatic artery catheters are removed. However, the venous and arterial sheaths and internal jugular catheter are not removed until coagulation normalizes. The patient is placed in a monitored setting for a minimum of $12 \mathrm{~h}$ and is maintained on bedrest for $4 \mathrm{~h}$ post-procedure. Postoperative laboratory studies are usually assessed daily while the patient is in the hospital, and once a patient's liver function tests and complete blood count stabilize, they are discharged. Labs are repeated within 5-7 days after discharge and weekly due to delayed hematologic changes secondary to melphalan exposure, which generally has a nadir of 7-10 days post-procedure [Figure 2].

\section{What is melphalan}

L-phenylalanine mustard (melphalan) is an alkylating agent. It has been attractive for use in PHP because as an agent used for regional therapy, its peak perfusate concentrations are 10 - to 100 -fold higher than maximally tolerated peak levels with systemic intravenous administration. ${ }^{[10]}$ Melphalan is active against both resting and rapidly dividing tumor cells. The maximum level of melphalan-induced DNA crosslinks is reached within $4 \mathrm{~h}$ of regional perfusion and declines thereafter. ${ }^{[11]}$ Side effects and toxicities observed from a Phase I trial are described in detail below.

\section{PHP vs. IHP}

There are some advantages to PHP when compared with IHP. Multiple infusions can be administered via PHP, which may improve the duration of responses compared to a single infusion using IHP. A percutaneous approach also avoids the morbidity of an open surgical procedure. However, the complications resulting from this type of procedure are those commonly associated with vascular procedures, including, but not limited to, hepatic artery dissection, hematoma, pseudoaneurysm, pneumothorax from line placement, and possible device failure. Specifically, deep venous thrombosis, heparin induced thrombocytopenia, anaphylaxis to protamine have been observed..$^{[8]}$ In comparison to PHP, IHP has the advantage of the ability to administer hyperthermic chemotherapy up to a temperature of $40{ }^{\circ} \mathrm{C}$, which would otherwise be fatal if systemically administered; this can be accomplished in IHP due to the complete surgical isolation of hepatic blood flow in a closed circuit. ${ }^{[2]}$

One must have experience with PHP as it can result in transient hemodynamic changes, such as decreased mean arterial blood pressure and venous return secondary to initiation of extracorporeal filtration and mechanical occlusion of the inferior vena cava. Acidosis has also been observed requiring the administration of intravenous sodium bicarbonate. ${ }^{[12]}$ Therefore, PHP must be done with a welltrained, experienced, and coordinated multidisciplinary team consisting of a vascular surgeon or interventional radiologist, anesthesiologist, and physicians that can safely manage the effects from the procedure and chemotherapy in a closely monitored setting.

\section{DATA AND OUTCOMES OF TRIALS}

\section{Phase I dose escalation trial}

The initial study evaluating the feasibility of hepatic arterial melphalan infusion using PHP for unresectable hepatic malignancies was completed by Pingpank et al..$^{[8]}$ The phase I study treated an initial cohort of 12 patients at $2.0 \mathrm{mg} / \mathrm{kg}$, followed by an additional 16 patients treated with escalating doses to the maximum tolerated dose (MTD) of $3.0 \mathrm{mg} / \mathrm{kg}$. A total of 78 treatments were administered to 28 patients. ${ }^{[8]}$ The histologies of patients with metastatic liver disease included: ocular melanoma, neuroendocrine neoplasms, colorectal cancer, cutaneous melanoma, adrenocortical carcinoma, pancreatic adenocarcinoma, retroperitoneal sarcoma, breast adenocarcinoma, and renal cell carcinoma. Three patients with unresectable primary hepatobiliary tumors also received treatment. At $3.5 \mathrm{mg} / \mathrm{kg}$, a dose limiting toxicity of neutropenia and/or thrombocytopenia was observed in 2 of 6 patients. Many patients who were treated experienced transient hepatic and systemic toxicities. 
Pharmacokinetic analysis revealed that there was no degradation of melphalan during the $30 \mathrm{~min}$ infusion and rapid intrahepatic clearance occurred within 10 min of completing the infusion. No renal, cardiac, or pulmonary complications were observed in patients after treatment with melphalan in PHP. The treatment course for this study was planned approximately every 4 to 6 weeks for a total of 4 treatments, and patients were required to recover from the previous treatment toxicity to grade II or less prior to embarking on the next perfusion. The investigators evaluated responses in the 27 evaluable patients using standard RECIST criteria. Reported antitumor activity included minor responses $(n=10)$, partial responses (PR) $(n=6)$ and complete responses (CR) $(n=2)$. At the time of the trial's publication the duration of responses included 2 PRs ongoing for 9 and 11 months and 2 CRs at 10 and 12 months. The overall radiographic objective response rate was found to be $30 \%$, and impressively in a subgroup of patients with ocular melanoma the overall objective response rate was found to be $50 \%$. The authors concluded that PHP, as a regional treatment of hepatic metastasis, can be safely performed with predictable and manageable toxicity.

\section{Moffitt cancer center experience}

Another trial described by Forster et al. ${ }^{[13]}$ retrospectively reviewed patients treated with PHP at their single institution over a 7 years period. The patients included those with unresectable melanoma or sarcoma hepatic metastases. Between 2008 and 2013, 10 patients were treated - a total of 27 PHP treatments were administered with the median number of treatments reported at 3 per patient. Nine of 10 $(90 \%)$ patients treated had stable disease (SD) or a PR, with a median partial response of a 33\% decrease in tumor burden from baseline. ${ }^{[13]}$ The median follow up for the evaluation was 11.5 months in which the hepatic progression free survival (hPFS) was 240 days. At last $60 \%$ of the patients treated at the institution died from their disease. The median overall survival from time of diagnosis of hepatic metastases was 12.6 months and from time of first PHP was 8.7 months. They also reported a median postoperative hospital stay of 3 days following PHP. The most common adverse event was myelosuppression which was treated on an outpatient basis. Seven of the patients in the cohort experienced a mild elevation in their serum troponin levels with the 1 patient having a value greater than $1.0 \mathrm{ng} / \mathrm{mL}$. There was no electrocardiography or echocardiographic evidence of myocardial ischemia, dyskinesia or dysfunction. The authors concluded from these results that for select patients with unresectable melanoma or sarcoma hepatic metastases, PHP is a safe and promising management option. ${ }^{[13]}$

\section{European experience}

Vogl et al. ${ }^{[14]}$ reported a European experience of patients with hepatic metastases treated with PHP using melphalan. Fourteen patients were treated between January 2012 and February 2013 at 2 centers with the following histologies: ocular or cutaneous melanoma, breast cancer, gastric cancer, and cholangiocarcinoma. These patients received $3.0 \mathrm{mg} / \mathrm{kg}$ of melphalan similar to the phase I trial reported by Pingpank et al ${ }^{[8]}$ The tumor response included $1 \mathrm{CR}$ seen in the cholangiocarcinoma patient, and 6 PRs (ocular melanoma: $n=3$, cutaneous melanoma: $n=3$ ). Stable disease was observed in 5 patients (ocular melanoma: $n=3$, breast cancer and gastric cancer). Toxicity was similar to that seen in previous series including melphalan-related thrombocytopenia, anemia and pancytopenia. In this series, second generation filters were used in a select number of patients. These filters are reported to have increased melphalan extraction efficiency. ${ }^{[15]}$ In the portion of patients treated with the second generation filters, the toxicity was found to be milder and patients experienced a faster recovery. ${ }^{[14]}$ Similar to other groups with experience in PHP, Vogl et al. ${ }^{[14]}$ concluded that PHP for non-resectable liver metastasis is a feasible treatment.

\section{Phase III multicenter trial}

These single-center phase I and II studies established the framework for a multicenter phase III trial with melphalan in 2005. Hughes et al. ${ }^{[16]}$ published results of the phase III, multicenter randomized trial comparing PHP with melphalan (PHP-Mel) to best alternative care (BAC) for patients with cutaneous or ocular melanoma metastatic to the liver. The trial accrued 93 patients between February 2006 and July 2009. Those enrolled were randomized to PHP-Mel $(n=44)$ or BAC $(n=49)$. Primary BAC treatment included systemic chemotherapy, chemoembolization, radioembolization, immunoembolization and supportive care. The trial design allowed for crossover to PHP-Mel for patients who experienced hepatic progression in the BAC arm, provided they still met enrollment criteria. The percutaneous procedure involved delivery of high dose melphalan directly to the liver via the hepatic artery over $30 \mathrm{~min}$. The initial dose of melphalan administered was $3.0 \mathrm{mg} / \mathrm{kg}$ based on ideal body weight. If a dose-limiting toxicity was encountered, the melphalan dose was decreased to $2.5 \mathrm{mg} / \mathrm{kg}$ in subsequent PHPs. Those randomized to PHP-Mel received treatment every 4-8 weeks when hematologic toxicity resolved to a grade 2 or less Patients were eligible to receive up to 6 PHP procedures in the absence of progressive disease..$^{[9]}$

The primary endpoint reported by Hughes et al. ${ }^{[16]}$ includes hPFS, with secondary endpoints including xPFS (date of randomization to the first observation of extrahepatic disease progression or death due to any cause), hepatic objective response (hOR), objective response rate (ORR), overall progression-free survival (oPFS), overall survival (OS), and safety. The results of the trial include a median hPFS in PHP-Mel of 7 months compared to 1.6 months in BAC. The median oPFS was 5.4 months and 1.6 months in PHP-Mel and BAC, respectively. The hOR for PHP-Mel was noted to be $36.4 \%$ with a SD rate of $52.3 \%$; hepatic disease control was observed in $75 \%$ of patients. The authors report a significant improvement in response favoring PHP-Mel patients including an ORR of 27.3\% (median duration 6.3 months) in the PHP-Mel group compared to $4.1 \%$ (median duration 3.7 months) in those who received BAC. There was no significant difference 
in OS observed between the 2 groups - the median OS of 10.6 months observed in PHP-Mel vs. 10.0 months in BAC was due to the built in crossover design. ${ }^{[16]}$

Hughes et al. ${ }^{[16]}$ described immediate peri-procedural events (within $72 \mathrm{~h}$ ) observed in $90 \%$ of PHP-Mel treated patients to include mostly self-limited thrombocytopenia and anemia. These events were attributed to platelet sequestration in the filters and/or hemodilution. The delayed post-procedural events, defined as occurring between 3 to 20 days after the melphalan exposure or until the next treatment cycle, were thought to be hematologic due to imperfect filtration. Neutropenia, thrombocytopenia and anemia were observed in most PHP-Mel patients and thought to be related to the effects of bone marrow suppression. Hyperbilirubinemia was observed in 10 patients. Some fatalities were observed on this trial and each death lead to further safety maneuvers in the development of improved filters. ${ }^{[15,16]}$

The authors concluded that the results of their phase III study demonstrate the efficacy of PHP-Mel. They report that the toxicity is significant but manageable in order to provide effective therapy for this select cohort of patients. Overall, given the improved hepatic PFS, oPFS, and hOR, Hughes et al. ${ }^{[16]}$ conclude that PHP with melphalan should provide a new treatment strategy for patients with unresectable metastatic melanoma to the liver.

\section{CONCLUSION}

PHP has been shown to be an innovative and promising technique for delivering regional chemotherapy to the liver. The evaluation of its use for different tumor histologies, has been, and continues to be studied in numerous trials. PHP has significant potential for the control of tumor burden in metastatic melanoma, particularly for ocular melanoma, which seems to be less responsive to checkpoint inhibition and other immunotherapies in comparison to cutaneous melanoma. ${ }^{[17]}$ The advantage of PHP lies in the ability to administer multiple therapies using a less invasive approach, in contrast to the laparotomy required for a single therapy with IHP. Currently, PHP in the United States is only available on study or compassionate use, however it does have the European mark and is being aggressively evaluated in seven different European countries. ${ }^{[14]}$ In the current landscape of liver directed therapy, PHP is a viable option for those with unresectable metastatic disease to the liver.

\section{Financial support and sponsorship}

This research was supported by the NIH grant: NCI 04-C-0273 (http:/grantome.com/grant/NIH/ZIA-BC011012-06).

\section{Conflicts of interest}

There are no conflicts of interest.

\section{REFERENCES}

1. Ruers T, Bleichrodt RP. Treatment of liver metastases, an update on the possibilities and results. Eur J Cancer 2002;38:1023-33.

2. Reddy SK, Kesmodel SB, Alexander HR Jr. Isolated hepatic perfusion for patients with liver metastases. Ther Adv Med Oncol 2014;6:180-94.

3. Alexander HR Jr, Butler CC. Development of isolated hepatic perfusion via the operative and percutaneous techniques for patients with isolated and unresectable liver metastases. Cancer J 2010;16:132-41.

4. Magge D, Choudry HA, Zeh HJ 3rd, Cunningham DE, Steel J, Holtzman MP, Jones HL, Pingpank JF, Bartlett DL, Zureikat AH. Outcome analysis of a decade-long experience of isolated hepatic perfusion for unresectable liver metastases at a single institution. Ann Surg 2014;259:953-9.

5. Lienard D, Ewalenko P, Delmotte JJ, Renard N, Lejeune FJ. High-dose recombinant tumor necrosis factor alpha in combination with interferon gamma and melphalan in isolation perfusion of the limbs for melanoma and sarcoma. J Clin Oncol 1992;10:52-60.

6. Ravikumar TS, Pizzorno G, Bodden W, Marsh J, Strair R, Pollack J, Hendler R, Hanna J, D’Andrea E. Percutaneous hepatic vein isolation and high-dose hepatic arterial infusion chemotherapy for unresectable liver tumors. J Clin Oncol 1994;12:2723-36.

7. Curley SA, Newman RA, Dougherty TB, Fuhrman GM, Stone DL, Mikolajek JA, Guercio S, Guercio A, Carrasco CH, Kuo MT, Hohn DC. Complete hepatic venous isolation and extracorporeal chemofiltration as treatment for human hepatocellular carcinoma: a phase I study. Ann Surg Oncol 1994; 1:389-99.

8. Pingpank JF, Libutti SK, Chang R, Wood BJ, Neeman Z, Kam AW, Figg WD, Zhai S, Beresneva T, Seidel GD, Alexander HR. Phase I study of hepatic arterial melphalan infusion and hepatic venous hemofiltration using percutaneously placed catheters in patients with unresectable hepatic malignancies. J Clin Oncol 2005;23:3465-74.

9. Pingpank JF, Hughes MS, Alexander HR, Faries MB, Zager JS, Royal R, Whitman ED, Nutting CW, Siskin GP, Agarwala SS. A phase III random assignment trial comparing percutaneous hepatic perfusion with melphalan (PHP-mel) to standard of care for patients with hepatic metastases from metastatic ocular or cutaneous melanoma. J Clin Oncol 2010;28 suppl:LBA8512.

10. Minor DR, Allen RE, Alberts D, Peng YM, Tardelli G, Hutchinson J. A clinical and pharmacokinetic study of isolated limb perfusion with heat and melphalan for melanoma. Cancer 1985;55:2638-44.

11. Parsons PG, Carter FB, Morrison L, Regius Mary Sister. Mechanism of melphalan resistance developed in vitro in human melanoma cells. Cancer Res 1981;41:1525-34.

12. Miao N, Pingpank JF, Alexander HR, Steinberg SM, Beresneva T, Quezado ZM. Percutaneous hepatic perfusion in patients with metastatic liver cancer: anesthetic, hemodynamic, and metabolic considerations. Ann Surg Oncol 2008;15:815-23.

13. Forster MR, Rashid OM, Perez MC, Choi J, Chaudhry T, Zager JS. Chemosaturation with percutaneous hepatic perfusion for unresectable metastatic melanoma or sarcoma to the liver: a single institution experience. J Surg Oncol 2014;109:434-9.

14. Vogl TJ, Zangos S, Scholtz JE, Schmitt F, Paetzold S, Trojan J, Orsi F, Lotz G, Ferrucci P. Chemosaturation with percutaneous hepatic perfusions of melphalan for hepatic metastases: experience from two European centers. Rofo 2014;186:937-44.

15. Moeslein FM, McAndrew EG, Appling WM, Hryniewich NE, Jarvis KD, Markos SM, Sheets TP, Uzgare RP, Johnston DS. Evaluation of Delcath Systems' Generation 2 (GEN 2) melphalan hemofiltration system in a porcine model of percutaneous hepatic perfusion. Cardiovasc Intervent Radiol 2014;37:763-9.

16. Hughes MS, Zager J, Faries M, Alexander HR, Royal RE, Wood B, Choi J, McCluskey K, Whitman E, Agarwala S, Siskin G, Nutting C, Toomey MA, Webb C, Beresnev T, Pingpank JF. Results of a randomized controlled multicenter phase III trial of percutaneous hepatic perfusion compared with best available care for patients with melanoma liver metastases. Ann Surg Oncol 2016;23:1309-19.

17. Niederkorn JY. Immune escape mechanisms of intraocular tumors. Prog Retin Eye Res 2009;28:329-47. 